\title{
Nonlinear evolution of dark matter and dark energy in the Chaplygin-gas cosmology
}

\author{
Neven Bilić†, Robert J Lindebaum $\ddagger$, Gary B Tupper§, and \\ Raoul D Viollier§ \\ † Rudjer Bošković Institute, P.O. Box 180, 10002 Zagreb, Croatia \\ ¥School of Chemical and Physical Sciences, University of Natal, Private Bag X01, \\ Scottsville 3209, South Africa \\ $\S$ Institute of Theoretical Physics and Astrophysics, Department of Physics, \\ University of Cape Town, Private Bag, Rondebosch 7701, South Africa \\ E-mail: bilic@thphys.irb.hr, lindebaumr@nu.ac.za, \\ viollier@physci.uct.ac.za
}

\begin{abstract}
The hypothesis that dark matter and dark energy are unified through the Chaplygin gas, an exotic fluid obeying $p=-A / \rho$, is reexamined. Using generalizations of the spherical model which incorporate effects of the acoustic horizon we show that an initially perturbative Chaplygin gas evolves into a mixed system containing cold dark matter like gravitational condensate.
\end{abstract}




\section{Introduction}

It has long been appreciated that there exists a dark-matter problem in that the nonrelativistic matter fraction $\Omega_{\mathrm{M}}$ of critical density, inferred from galactic rotation curves, cluster stability, and peculiar velocities, far exceeds the ordinary baryonic matter fraction $\Omega_{B} \simeq 0.04$ provided by nucleosynthesis, but falls short of the standard inflationary prediction of unity [1]. Observations of high-redshift supernova [2] and microwave background anisotropies [3, 4] have now settled the case for a flat universe with the dominant component dubbed 'dark energy' possessing appreciable negative pressure, such that the ratio $w \equiv p / \rho$ is less than -0.8 . The most conservative reading of the WMAP results [4] is that $\Omega_{\mathrm{CDM}} \simeq 0.23$ is provided by traditional cold dark matter $(\mathrm{CDM})$ candidates, while the balance resides in a cosmological constant $\Lambda$ with the corresponding density fraction $\Omega_{\Lambda} \simeq 0.73$. The inherent fine-tuning and coincidence problems of the $\Lambda \mathrm{CDM}$ model are somewhat ameliorated in quintessence models [5], which replace $\Lambda$ by an evolving scalar field. However, like its predecessor, a quintessenceCDM model assumes that dark matter and dark energy are distinct entities. For a recent review on the most popular dark-matter and dark-energy models, see [6].

A more liberal interpretation is that these data may tell us something profound about the space-time we inhabit, dark matter/energy being different manifestations of a common structure encoded in the dark sector's $w$ [7]. An interesting possibility becomes evident upon recalling that the average density in a virialized halo is more than several hundred times higher than the critical density, suggesting a density dependent $w=w(\rho)$ with $w \simeq 0$ for $\rho \gg \rho_{\text {cr }}, w \sim-1$ for $\rho \sim \rho_{\text {cr }}$ and the identification of "dark matter" with objects which became nonlinear at high redshift. The general class of models in which a unification of dark matter and dark energy is achieved through a single entity is often referred to as quartessence [8, 9]. Among other scenarios of unification that

have recently been suggested, interesting attempts are based on the so-called $k$-essence [10, 11], a scalar field with noncanonical kinetic terms which was first introduced as a model for inflation 12 .

Perhaps the simplest scenario in which dark matter and dark energy are different manifestations of a single substance may be realized through the Chaplygin gas, an exotic fluid obeying

$$
p=-\frac{A}{\rho},
$$

which has been extensively studied for its mathematical properties [13]. The cosmological potential of equation (11) was first noted by Kamenshchik et al [14] who observed that integrating the energy conservation equation in a homogeneous model led to

$$
\rho(a)=\sqrt{A+\frac{B}{a^{6}}},
$$

where $a$ is the scale factor normalized to unity today and $B$ an integration constant. Thus, the Chaplygin gas interpolates between matter, $\rho \sim a^{-3}$, at high redshift and a 
cosmological constant like $\rho \sim \sqrt{A}$ as $a$ tends to infinity.

Of particular interest is that equation (11) is obtained [13, 15, 16] from the Lagrangian

$$
\mathcal{L}=-\sqrt{A} \sqrt{1-g^{\mu \nu} \theta_{, \mu} \theta_{, \nu}}
$$

which may be seen by evaluating the stress-energy tensor $T_{\mu \nu}$ and introducing $u_{\mu}=$ $\theta_{, \mu} / \sqrt{g^{\alpha \beta} \theta_{, \alpha} \theta_{, \beta}}$ for the four-velocity and $\rho=\sqrt{A} / \sqrt{1-g^{\mu \nu} \theta_{, \mu} \theta_{, \nu}}$ for the energy density. One recognizes $\mathcal{L}$ as a Lagrangian of the Born-Infeld type familiar in the $D$ brane constructions of string/ $M$ theory [13]. Geometrically, $\mathcal{L}$ describes space-time as a $3+1$ brane in a $4+1$ bulk via the embedding coordinate $X^{5}=\theta$. The same Lagrangian appears as the leading term in Sundrum's [17] effective field theory approach to large extra dimensions; for one such extra dimension, $0 \leq \theta \leq \ell_{5}$, lowering the Planck scale to the intermediate scale of about $10^{11} \mathrm{GeV}$, one estimates $A^{1 / 8} \sim \ell_{5}^{-1} \sim \mathrm{meV}$ [16].

The Born-Infeld Lagrangian (3) is a special case of the string-theory inspired tachyon Lagrangian [18] in which the constant $\sqrt{A}$ is replaced by a potential $V(\theta)$. Unfortunately, tachyon models encounter the problem that, generically, $V(0)$ is a maximum, so the tachyon field rolls quickly towards the minimum $V(\infty)=0$ thereby driving $w=\dot{\theta}^{2}-1 \rightarrow 0$ to leave only a dust component [19]. However, the situation may be quite different in inhomogeneous tachyon cosmology [20].

It is possible to define a 'generalized Chaplygin gas' 21] through

$$
p=-A / \rho^{\alpha},
$$

where $\alpha \geq 0$. In this case, the analogue of equation (3) lacks any geometrical interpretation, but equation (44) can be obtained from a moving brane in Schwarzschildanti-de-Sitter bulk 22]. In the limit $\alpha \rightarrow 0$, one approaches a model equivalent to $\Lambda \mathrm{CDM}$ in the background equations, although the perturbation equations yield a different power spectrum [23].

All models that unify dark matter and dark energy face the problem of nonvanishing sound speed and the well-known Jeans instability. A fluid with a nonzero sound speed has a characteristic scale below which the pressure effectively opposes gravity. Hence the perturbations of the scale smaller than the sonic horizon will be prevented from growing. In the Chaplygin-gas model, the sound speed is small at early times but is significant at the crossover region and hence the small scale perturbations are dumped. Indeed, it has been demonstrated that the purely perturbative Chaplygin-gas model [24, 25] is in severe conflict with the cosmic microwave background (CMB) angular power spectrum [26, 27. and the mass power spectrum [28. However, it has recently been pointed out that if nonadiabatic perturbations are allowed, the Chaplygin-gas model may be compatible with the $2 \mathrm{df}$ mass power spectrum [29].

A more general situation where the Chaplygin gas is mixed with CDM is considered in a number of papers [30, 31, 32, 33, 34, 35, 36, 37. In this case, the Chaplygin gas simply plays the role of dark energy. In keeping with the quartessence philosophy it would be preferred if cold dark matter could be replaced by droplets of Chaplygin condensate. Homogeneous world models containing a mixture of cold dark matter 
and Chaplygin gas have been successfully confronted with lensing statistics [30, 31] as well as with supernova and other tests [32, 33. Bean and Doré [34 and similarly Amendola et al [35] have examined a mixture of CDM and the generalized Chaplygin gas against supernova, large-scale structure, and CMB constraints and demonstrated that a thorough likelihood analysis favors the limit $\alpha \rightarrow 0$, i.e., the equivalent $\Lambda \mathrm{CDM}$ model. In both papers it has been concluded that the standard Chaplygin gas is ruled out as a candidate for dark energy. However, a recent analysis [37, 38] of the supernova data seems to indicate that the generalized Chaplygin gas with $\alpha \geq 1$ is favored over the $\alpha \rightarrow 0$ model.

In spite of these somewhat discouraging results we share the opinion of Gorini et al [39] that the Chaplygin gas still deserves further investigation and that a thorough investigation of the nonlinear regime of the growth of inhomogeneities is needed for a definite conclusion concerning the compatibility of the Chaplygin gas cosmologies with the observable large-scale structure of the universe. On the basis of an extended spherical model which includes the sonic horizon effects, in this paper we show that a fraction of the Chaplygin gas condensates and never reaches a stage where its properties change from dark-matter-like to dark-energy-like. Hence, at the crossover region a fraction of the gas behaves effectively as dark matter and the rest as dark energy.

We organize the paper as follows. In section 2 we discuss the nonlinear evolution of perturbation in the Newtonian limit by generalizing the spherical model. Summary and conclusions are given in section 3 .

\section{Condensate Formation}

To be able to claim that the Chaplygin gas (or any other candidate) actually achieves unification, one must be assured that initial perturbations can evolve into a deeply nonlinear regime to form a gravitational condensate of superparticles that can play the role of cold dark matter. In [15, 16] this was inferred on the basis of the Zel'dovich approximation [40]. In comoving coordinates, the solution for the inhomogeneous Chaplygin-gas cosmology is

$$
\rho=\sqrt{A+\frac{B}{\gamma}} .
$$

Here $\gamma$ is the determinant of the induced spatial metric tensor $\gamma_{i j}=g_{i 0} g_{j 0} / g_{00}-g_{i j}$ and $B$ can be taken as constant on the scales of interest. The generalization (15) of equation (2) allows us to implement the geometric version of the Zel'dovich approximation: the transformation from Euler to Lagrange (comoving) coordinates induces $\gamma_{i j}$ as

$$
\gamma_{i j}=\delta_{k l} D_{i}{ }^{k} D_{j}{ }^{l}, \quad D_{i}{ }^{j}=a\left(\delta_{i}{ }^{j}-b \varphi_{, i}{ }^{j}\right),
$$

where $D_{i}{ }^{j}$ is the deformation tensor, $\varphi$ is the velocity potential, and the quantity $b=b(t)$ describes the evolution of the perturbation. The Zel'dovich approximation offers a means of extrapolation into the nonperturbative regime via equation (15) and

$$
\gamma=a^{6}\left(1-\lambda_{1} b\right)^{2}\left(1-\lambda_{2} b\right)^{2}\left(1-\lambda_{3} b\right)^{2}
$$


where the $\lambda_{i}$ are the eigenvalues of $\varphi_{, i}{ }^{j}$. When one (or more) of the $\lambda$ 's is (are) positive, a caustic forms on which $\gamma \rightarrow 0$ and $w \rightarrow 0$, i.e., at the locations where structure forms the Chaplygin gas behaves as dark matter. Conversely, when all of the $\lambda$ 's are negative, a void forms, $\rho$ is driven to its limiting value $\sqrt{A}$, and the Chaplygin gas behaves as dark energy, driving accelerated expansion.

For the issue at hand, the usual Zel'dovich approximation has the shortcoming that the effects of finite sound speed are neglected. Since the structure formation occurs in the decelerating phase, we can address the question in the Newtonian limit $p \ll \rho$ by generalizing the spherical model. In the case of vanishing shear and rotation, the continuity and Euler-Poisson equations become

$$
\begin{aligned}
& \dot{\rho}+3 \mathcal{H} \rho=0, \\
& 3 \dot{\mathcal{H}}+3 \mathcal{H}^{2}+4 \pi G \rho+\frac{\partial}{\partial r_{i}}\left(\frac{c_{s}^{2}}{\rho} \frac{\partial}{\partial r_{i}} \rho\right)=0,
\end{aligned}
$$

where $c_{s}$ is the adiabatic speed of sound given by

$$
c_{s}^{2}=\frac{\partial p}{\partial \rho}=\frac{A}{\rho^{2}}
$$

$\mathcal{H}$ is the local Hubble parameter, and the time derivative is at the fixed Lagrangian coordinate $\vec{r}$. It is convenient to introduce the comoving coordinates $\vec{x}=\vec{r} / a$. Then writing

$$
\begin{aligned}
& \rho=\bar{\rho}(1+\delta), \\
& \mathcal{H}=H+\delta \mathcal{H},
\end{aligned}
$$

subtracting the background, eliminating $\delta \mathcal{H}$ and $\delta \dot{\mathcal{H}}$, and changing the variables from $t$ to $a$ we find

$$
a^{2} \delta^{\prime \prime}+\frac{3}{2} a \delta^{\prime}-\frac{3}{2} \delta(1+\delta)-\frac{4}{3} \frac{\left(a \delta^{\prime}\right)^{2}}{1+\delta}-\frac{1+\delta}{a^{2} H^{2}} \frac{\partial}{\partial x_{i}}\left(\frac{c_{s}^{2}}{1+\delta} \frac{\partial \delta}{\partial x_{i}}\right)=0
$$

where' denotes the derivative with respect to $a$. To solve this partial differential equation as it stands, one would need a rather involved numerical computation. Instead, we proceed first by discussing the linear approximation of (13) and then by solving the nonlinear problem imposing certain restrictions on the solution.

First, consider the linear approximation which has been discussed in detail by Fabris et al [41]. Keeping only the terms linear in $\delta, \delta^{\prime}$, and $\delta^{\prime \prime}$ and using the expansion

$$
\delta(a, \vec{x})=\sum_{k} \delta_{\text {pert }}(k, a) e^{i \vec{k} \vec{x}}
$$

one obtains an explicit solution for the perturbative density contrast which may be expressed as

$$
\delta_{\text {pert }}(k, a) \propto a^{-1 / 4} J_{5 / 14}\left(d_{\mathrm{s}} k\right) .
$$


Here $J_{\nu}(z)$ is the Bessel function, $k$ the comoving wave number, and $d_{\mathrm{s}}$ the comoving sonic horizon given by

$$
d_{\mathrm{s}}=\int_{0}^{a} \frac{\bar{c}_{s} d a}{a^{2} H}=\frac{2}{7} \frac{\left(1-\Omega^{2}\right)^{1 / 2}}{\Omega^{3 / 2}} \frac{a^{7 / 2}}{H_{0}},
$$

with $\bar{c}_{s}=\sqrt{A} / \bar{\rho}$ and the equivalent matter fraction

$$
\Omega=\sqrt{B /(A+B)}=\sqrt{B} / \rho_{\text {cr }} .
$$

Thus, for $d_{\mathrm{s}} k \ll 1, \delta_{\text {pert }} \sim a$, but for $d_{\mathrm{s}} k \gg 1, \delta_{\text {pert }}$ undergoes damped oscillations. Similar results have been obtained numerically in the relativistic case [42].

Next, consider the nonlinear evolution. To simplify equation (13), it seems reasonable to make the following restriction: we take $\delta(t, \vec{x})$ to be of fixed shape, wave number dependent size, and time dependent amplitude, then evaluate the comoving coordinate derivatives at the origin. That is to say, we retain the spirit of the spherical dust model where each comoving coordinate point is treated as an independent origin, but allow for the local coupling of the inhomogeneity curvature to sound waves. A convenient choice is the spherical lump

$$
\delta(a, \vec{x})=\delta_{R}(a) f(x / R),
$$

where $f(z)$ is an arbitrary function satisfying $f^{\prime}(0)=1$ and $f^{\prime \prime}(0)<0$. For simplicity, we take $f(z)=\exp \left(-z^{2} / 2\right)$. Evaluating (13) at the origin, we obtain an ordinary differential equation for $\delta_{R}$

$$
a^{2} \delta_{R}^{\prime \prime}+\frac{3}{2} a \delta_{R}^{\prime}-\frac{3}{2} \delta_{R}\left(1+\delta_{R}\right)-\frac{4}{3} \frac{\left(a \delta_{R}^{\prime}\right)^{2}}{1+\delta_{R}}+\frac{49}{4}\left(\frac{a}{a_{R}}\right)^{7} \frac{\delta_{R}}{\left(1+\delta_{R}\right)^{2}}=0,
$$

where

$$
a_{R}^{-1} \equiv\left(d_{\mathrm{s}} \sqrt{3} / R\right)^{2 / 7} a^{-1}=8.06\left(1-\Omega^{2}\right)^{1 / 7} \Omega^{-3 / 7}\left(R^{-1} h^{-1} \mathrm{Mpc}\right)^{2 / 7} .
$$

The recommendations of equation (19) are that it

(i) reproduces equation (15) at linear order and

(ii) extends the spherical dust model by incorporating the Jeans length through the last term.

To gain some perspective, with WMAP fixing $a_{\mathrm{eq}}=3 \cdot 10^{-4}$ and reionization at a redshift of 20 , initial perturbations must satisfy $\delta\left(a_{\text {eq }}\right) \gtrsim 6 \cdot 10^{-3}$ on mass scales $M \lesssim 10^{10} M_{\odot}$ even for $\Lambda \mathrm{CDM}$. Taking $\Omega=0.27$ and $h=0.71$, we find $a_{R}^{-1}=$ $15.4\left(R^{-1} \mathrm{Mpc}\right)^{2 / 7}$, which coincides with $1+z_{\text {reion }}=21$ at $R=0.338 \mathrm{Mpc}$ corresponding to a protogalaxy mass $M=2.3 \cdot 10^{10} M_{\odot}$.

In figure 1 we show the evolution of two initial perturbations from the radiationmatter equality for $a_{R}=1 / 21$. In contrast to linear theory, where for any $R$ the acoustic horizon will eventually stop $\delta_{R}$ from growing irrespective of the initial value of the perturbation, here, for initial $\delta_{R}\left(a_{\mathrm{dec}}\right)$ above a certain threshold, $\delta_{R}(a) \rightarrow \infty$ at finite $a$ just as in the dust model. Conversely, at sufficiently small $\delta_{R}\left(a_{\mathrm{dec}}\right)$, the acoustic horizon can stop $\delta_{R}(a)$ from growing even in a mildly nonlinear regime. Figure 2 shows 




Figure 1. Evolution of $\delta_{R}(a)$ in the spherical model, equation (19), from $a_{\mathrm{eq}}=$ $3 \times 10^{-4}$ for $a_{R}=0.0476, \delta_{R}\left(a_{\mathrm{eq}}\right)=0.004($ solid $)$ and $\delta_{R}\left(a_{\mathrm{eq}}\right)=0.005($ dashed $)$.

how the threshold $\delta_{R}\left(a_{\mathrm{dec}}\right)$ divides the two regimes depending on the comoving scale $R$. Qualitatively similar conclusions have been reached in a different way by Avelino et al [43].

One question is how accurate is the approximation (18) which assumes that the comoving size is time independent? To check this, we have relaxed the assumption (18) by allowing the second derivative of $f$ at the origin to depend on $a$, i.e., the initial Gaussian perturbation to have a variable width. Evolution of the width is then described by a second-order differential equation coupled to equation (19). By solving this system of coupled equations we find a slight change of the behavior of $\delta_{R}$ in the linear regime but no significant change of the linear/nonlinear boundary depicted in figure 2 .

The crucial question is what fraction of Chaplygin gas goes into condensate. Indeed, in [44] we have noted that if this were $92 \%$ as given by the geometric Zel'dovich approximation, the CMB and the mass power spectrum would be reproduced. To answer this quantitatively, we adopt the Press-Schechter approach [45]. Assuming $\delta_{R}\left(a_{\mathrm{dec}}\right)$ is given by a Gaussian random field with dispersion $\sigma(R)$, and including the notorious factor of 2 to account for the cloud in cloud problem, the condensate fraction at a scale $R$ is given by

$$
F(R)=2 \int_{\delta_{c}(R)}^{\infty} \frac{d \delta}{\sqrt{2 \pi} \sigma(R)} \exp \left(-\frac{\delta^{2}}{2 \sigma^{2}(R)}\right)=\operatorname{erfc}\left(\frac{\delta_{c}(R)}{\sqrt{2} \sigma(R)}\right),
$$






Figure 2. Initial value $\delta_{R}\left(a_{\mathrm{dec}}\right)$ versus $R$ for $\Omega=0.27$ and $h=0.71$. The threshold $\delta_{c}\left(a_{\mathrm{dec}}\right)$ is shown by the line separating the two regimes. The dashed line gives $\sigma(R)$ calculated using the concordance model.

where $\delta_{c}(R)$ is the threshold $\delta_{c}\left(a_{\mathrm{dec}}\right)$ depicted in figure 2. In figure 2 we also exhibit the dispersion

$$
\sigma^{2}(R)=\int_{0}^{\infty} \frac{d k}{k} \exp \left(-k^{2} R^{2}\right) \Delta^{2}\left(k, a_{\mathrm{dec}}\right)
$$

calculated using $f$ above as the window function and the concordance model 4] variance

$$
\Delta^{2}(k, a)=\text { const }\left(\frac{k}{a H}\right)^{4} T^{2}(k)\left(\frac{k}{7.5 a_{0} H_{0}}\right)^{n-1} .
$$

In figure 3 we present $F(R)$ calculated using (21)-(23) with const $=7.11 \times 10^{-9}$, the spectral index $n=1.02$, and the parametrization of Bardeen et al [46] for the transfer function $T(k)$ with $\Omega_{\mathrm{B}}=0.04$. The parameters are fixed by fitting (23) to the $2 \mathrm{dFGRS}$ power spectrum data [4]. Our result, in qualitative agreement with that of Avelino et al 43], demonstrates that the collapse fraction is less than $1 \%$, far too small to affect the conclusions of [27, 28].

\section{Summary and Conclusions}

Thus we have found that nonlinear condensate, while present, is insufficient to save the simple Chaplygin gas. In effect, the model is a victim of the radiation dominated phase which turns the Harrison-Zel'dovich spectrum $\delta_{k} \sim k^{1 / 2}$ to $\delta_{k} \sim k^{-3 / 2}$ at $R_{\text {eq }} \simeq 26 \mathrm{Mpc}$. In the pure Chaplygin-gas universe there would inevitably be insufficient small scale 


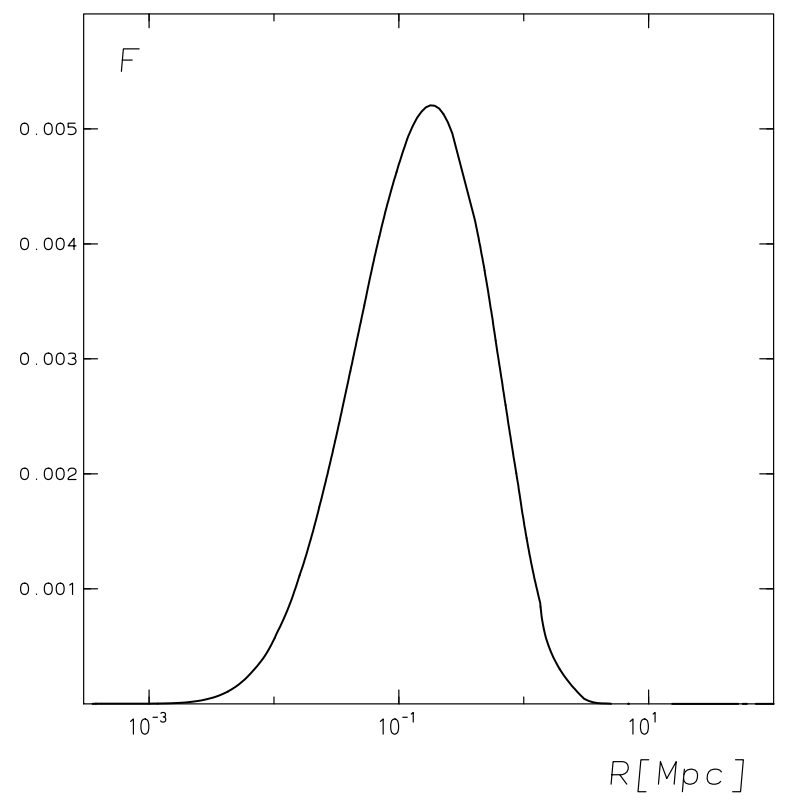

Figure 3. Fraction of Chaplygin gas in collapsed objects using $\delta_{c}(R)$ and $\sigma(R)$ from figure 2

power to drive condensation. This is a more definitive, if pessimistic, statement than [43] because we have actually followed the perturbations into the fully nonlinear regime. Pragmatically, one can follow [7] by introducing entropy perturbations to make the effective speed of sound vanish, even in the nonperturbative regime [29]. Caution should, however, be taken that imposing $\delta p=0$ as an initial condition does not guarantee its propagation except in the long-wavelength limit [48] where there is no problem in any case. More important is that the negative results were drawn without exploiting the braneworld connection of equation (31): in braneworld models (for a recent review, see [49]) the Einstein equations are modified, e.g., by dark radiation and hence so are equations (89). From a different perspective, similar changes are brought about by the radion mode [50] which gives a scalar-tensor gravity.

An interesting idea how to circumvent the difficulties with structure formation in the Chaplygin gas model has recently been put forward by Bento et al [51]. Following [20], they have identified $p=-\rho_{\Lambda}$ and $\rho_{\mathrm{dm}}=\rho+p$, so that the energy-momentum tensor appears as a combination of dark matter $\rho_{\mathrm{dm}}$ and the cosmological "constant" $\Lambda$. Still, $T_{\nu ; \mu}^{\mu}=0$ supplies the continuity and Euler equations while $\rho+3 p=\rho_{\mathrm{dm}}-2 \rho_{\Lambda}$ sources the gravitational potential. In particular, $\left(\partial \rho_{\Lambda} / \partial r_{i}\right) / \rho_{\mathrm{dm}}$ remains in the Euler equation. Since $|p| \ll \rho \simeq \rho_{\mathrm{dm}}$ in the Newtonian context, and $c_{\mathrm{dm}}^{2}=-\partial \rho_{\Lambda} / \partial \rho_{\mathrm{dm}}=$ $c_{s}^{2} /\left(1+c_{s}^{2}\right) \simeq c_{s}^{2}$, there is no modification to equations (8) and (9), or the conclusions on structure formation. The reason Bento et al [51] conclude this bookkeeping allows $\delta_{\mathrm{dm}}$ to grow unimpeded is that they have taken over the equations of Arcuri and Waga [52], wherein $\Lambda$ is assumed time dependent but homogeneous - $c f$ equation (14) therein. 
Here, instead, $\Lambda$ homogeneous implies $\rho_{\mathrm{dm}}$ homogeneous, i.e. $\delta_{\mathrm{dm}}=0$.

Our prognosis is then that the crisis in quartessence cosmology is akin to that which existed in electroweak gauge theory before the Higgs mechanism. The Chaplygin-gas model is not so much wrong as incomplete.

\section{Acknowledgments}

Two of us, GBT and RDV wish to thank Jihn Kim for many enlightening discussions on the braneworld aspects of the Chaplygin gas. This research is in part supported by the Foundation for Fundamental Research (FFR) grant number PHY99-1241 and the

Research Committee of the University of Cape Town. The work of NB is supported in part by the Ministry of Science and Technology of the Republic of Croatia under Contract No. 0098002.

\section{References}

[1] Turner M S, Steigman G and Krauss L 1984 Phys. Rev. Lett. 522090 Peebles P J E 1984 Astrophys. J. 284439

[2] Perlmutter S et al 1999 Astrophys. J. 517565

Riess A G et al 1998 Astron. J. 1161009

[3] Halverson N W et al 2000 Astrophys. J. 56838

Netherfield C B et al 2002 Astrophys. J. 571604

[4] Bennett C L et al 2003 Astrophys. J. Suppl. 1481 (Bennett C L et al 2003 Preprint astro-ph/0302207)

Spergel D N et al 2003 Astrophys. J. Suppl. 148175 (Spergel D N et al 2003 Preprint astro-ph/0302209)

[5] For a review see: Peebles P J E and Ratra B 2003 Rev. Mod. Phys. 75599

[6] Sahni V 2004 Preprint astro-ph/0403324

[7] Hu W 1998 Astrophys. J. 306485

[8] Makler M, de Oliveira S Q and Waga I 2003 Phis. Lett. B 5551

[9] Reis R R R, Makler M and Waga I 2004 Phys. Rev. D 69101301

[10] Chimento L P 2004 Phys. Rev. D 69123517

[11] Scherrer R J 2004 Phys. Rev. Lett. 93011301

[12] Armendariz-Picon C, Damour T and Mukhanov V 1999 Phys. Lett. B 458209

[13] Jackiw R 2002 Lectures on fluid dynamics. A Particle theorist's view of supersymmetric, nonAbelian, noncommutative fluid mechanics and d-branes (New-York: Springer-Verlag)

[14] Kamenshchik A, Moschella U and Pasquier V 2001 Phys. Lett. B 511265

[15] Bilić N, Tupper G B and Viollier R D 2002 Phys. Lett. B 53517

[16] Bilić N, Tupper G B and Viollier R D 2002 Proc. Int. Conf. in Dark Matter in Astro- and Particle Physics, DARK 2002 ed Klapdor-Kleingrothaus H V and Viollier R D (Berlin, Heidelberg: Springer-Verlag) (Bilić N, Tupper G B and Viollier R D 2002 Preprint astro-ph/0207423)

[17] Sundrum R 1999 Phys. Rev. D 59085009

[18] Sen A 2002 Mod. Phys. Lett. A 171797

Sen A 2002 J. High Energy Phys. JHEP04(2002)048

Sen A 2002 J. High Energy Phys. JHEP07(2002)065

[19] Gibbons G W 2003 Class. Quant. Grav. 20 S321

[20] Padmanabhan T and Roy Choudhury T 2002 Phys. Rev. D 66081301

[21] Bento M C, Bertolami O and Sen A A 2002 Phys. Rev. D 66043507 
[22] Neves R and Vas C 2003 Phys. Lett. B 568153

[23] Fabris J C, Gonçalves S V B and de Sá Ribeiro R 2004 Gen. Rel. Grav. 36211

[24] Cunha J V, Alcaniz J S and Lima J A S 2004 Phys. Rev. D 69083501

[25] Makler M, de Oliveira S Q and Waga I 2003 Phys. Rev. D 68123521

[26] Bento M C, Bertolami O and Sen A A 2003 Phys. Rev. D 67063003

[27] Carturan P and Finelli F 2003 Phys. Rev. D 68103501

[28] Sandvik H B, Tegmark M, Zaldarriaga M and Waga I 2004 Phys. Rev. D 69123524

[29] Reis R R R, Waga I, Calvão M O and Joŕas S E 2003 Phys. Rev. D 68061302

Reis R R R, Makler M and Waga I 2004 Phys. Rev. D 69101301

[30] Dev A, Alcaniz J S and Jain D 2003 Phys. Rev. D 67023515

[31] Dev A, Jain D and Alcaniz J S 2004 Astron. Astrophys. 417847

[32] Avelino P P, Beça L M G, de Carvalho J P M, Martins C J A P and Pinto P 2003 Phys. Rev. D 67023511

[33] Alcaniz J S, Jain D and Dev A 2003 Phys. Rev. D 67043514

[34] Bean R and Doré 02003 Phys. Rev. D 68023515

[35] Amendola L, Finelli F, Burigana C and Carturan D 2003 J. Cosmology Astroparticle Phys. JCAP07(2003)005

[36] Multamäki T, Manera M and Gaztañaga E 2004 Phys. Rev. D 69023004

[37] Bertolami O, Sen A A, Sen S and Silva P T 2004 MNRAS 353329

[38] Bertolami O 2004 Preprint astro-ph/0403310

[39] Gorini V, Kamenshchik A, Moschella U and Pasquier V 2004 Preprint gr-qc/0403062

[40] Zel'dovich Ya B 1970 Astron. Astrophys. 584

[41] Fabris J C, Gonçalves S V B and de Souza P E 2002 Gen. Rel. Grav. 3453

[42] Fabris J C, Gonçalves S V B and de Souza P E 2002 Gen. Rel. Grav. 342111

[43] Avelino P P, Beça L M G, de Carvalho J P M, Martins C J A P and Copeland E J 2004 Phys. Rev. D 69041301

[44] Bilic N, Lindebaum R J, Tupper G B and Viollier R D 2003 Preprint astro-ph/0310181, tp appear in Proc. BLOIS 2003

[45] Press W H and Schechter P 1974 Astrophys. J. 187425

[46] Bardeen J M, Bond J R, Kaiser N and Szalay A S 1986 Astrophys. J. 30415

[47] Percival W J et al 2001 MNRAS 3271297

[48] Abramo L R W, private communication

[49] Maartens R 2004 Living Rev. Rel. 71

[50] Kim J E, Tupper G B and Viollier R D 2004 Phys. Lett. B 593209

[51] Bento M C, Bertolami O and Sen A A 2004 Preprint astro-ph/0407239

[52] Arcuri R C and Waga I 1994 Phys. Rev. D 502928 